\title{
Introductory comments
}

\section{Commentaires préliminaires}

$\mathrm{T}_{5}^{\mathrm{h}}$ his is a special issue commemorating the 50th anniversary of the Canadian Thoracic Society (CTS). This spring, I was approached by Peter Warren, who indicated that he and the current CTS president, Darcy Marciniuk, believed that such a commemorative issue would be a good thing and that it should be about Canadian contributions to the field of respiratory medicine over the past 50 years.

I believe that this was a really good idea, because if there is one area where for 50 years Canada has 'punched above its weight', it is in fact respiratory medicine. Moreover, many of the main

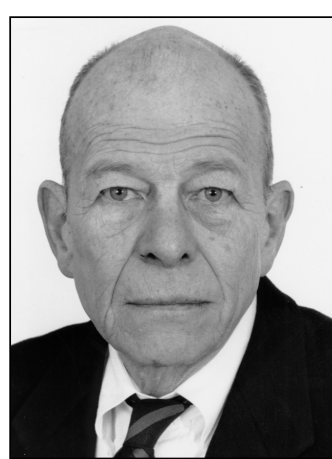

Nick R Anthonisen contributors to our glory are still active and productive. The idea was to get these distinguished physicians to each write a brief chapter on a topic of his or her choice, given some broad limitations. The time line was short: less than two months from the time a contributor assented to the deadline for the finished project. I contacted the potential contributors to ask them to do this; to my surprise and gratification, all accepted.

Furthermore, nearly all of them met the deadline, and the content of the present issue was generated.

The present issue boasts an all-star roster of authors, all writing on a subject dear to their heart. They illustrate very well the remarkable contributions that our community has made to the current state of the art. Both the CTS and I are profoundly grateful to them.

Nick R Anthonisen MD Editor-in-Chief, Canadian Respiratory Journal
Tous lisez un numéro spécial pour célébrer le 50e anniversaire de la Société canadienne de thoracologie (SCT). Ce printemps, Peter Warren a communiqué avec moi et m'a dit que lui et le président de la SCT, Darcy Marciniuk, pensaient qu'il serait bon de publier un tel numéro commémoratif et de le consacrer à l'apport des Canadiens à la médecine respiratoire depuis 50 ans.

Je trouve que c'était une excellente idée, parce que s'il y a un domaine où le Canada « a joué dans la cour des grands » depuis 50 ans, c'est bien la médecine respiratoire. De plus, bon nombre des principaux collaborateurs à notre gloire sont encore actifs et productifs. Le principe, c'était de demander à ces médecins émérites d'écrire chacun un bref chapitre sur le sujet de leur choix, dans un cadre de directives générales. L'échéancier était court : moins de deux mois entre l'acceptation et la remise du projet définitif. J'ai pris contact avec les collaborateurs potentiels pour les solliciter. À ma grande surprise et à mon grand plaisir, ils ont tous accepté.

De plus, ils ont presque tous respecté l'échéance, et le contenu du présent numéro a pu être produit.

Le présent numéro met en valeur un groupe d'auteurs étoiles qui écrivent tous sur un sujet qui leur tient à cœur. Ils illustrent à merveille l'apport remarquable de notre communauté à l'état actuel des connaissances. La SCT et moi leur en sommes profondément reconnaissants.

Nick R. Anthonisen, M.D. Rédacteur en chef du Journal canadien de pneumologie 


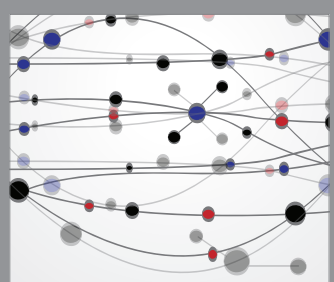

The Scientific World Journal
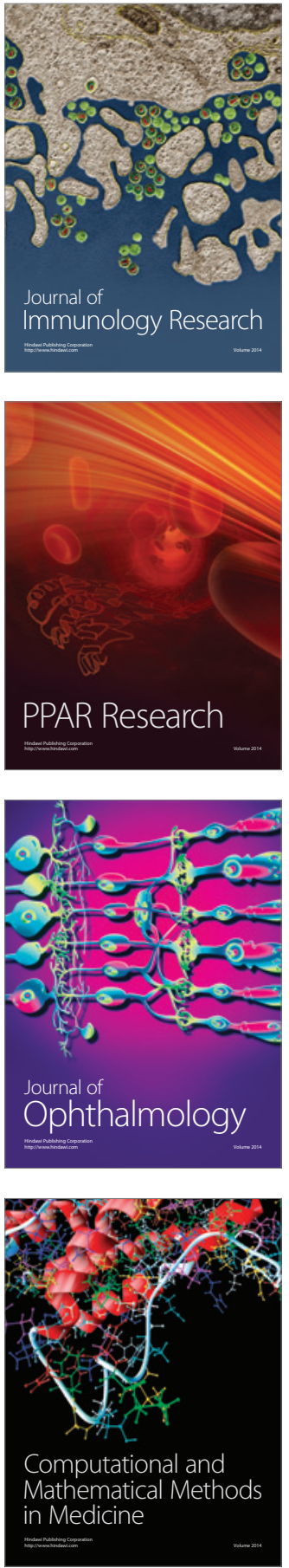

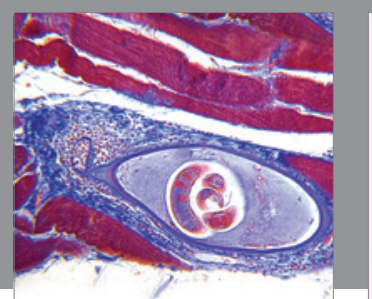

Gastroenterology Research and Practice

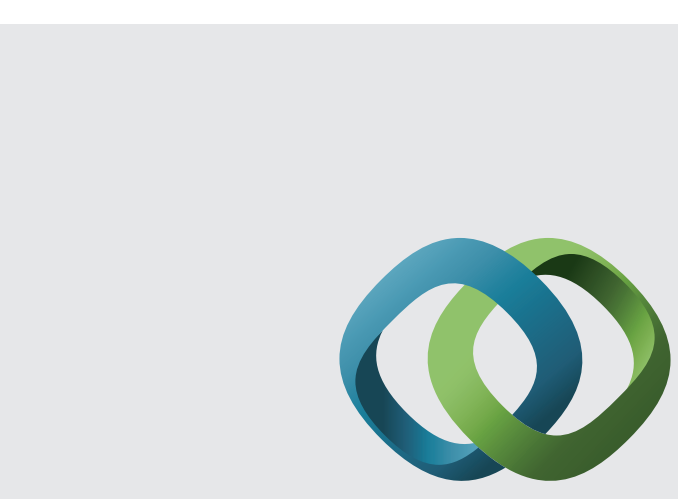

\section{Hindawi}

Submit your manuscripts at

http://www.hindawi.com
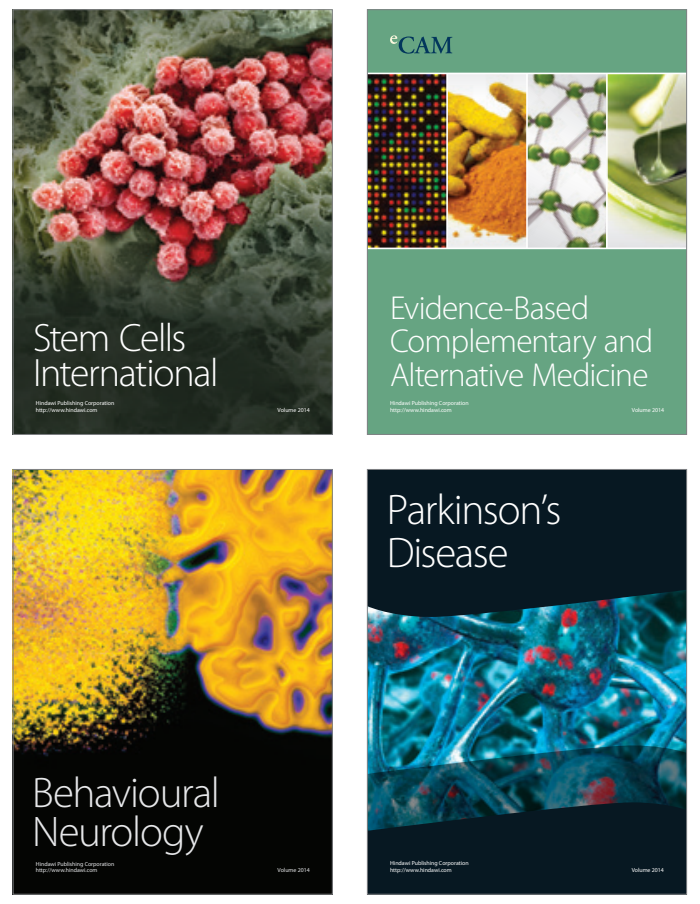
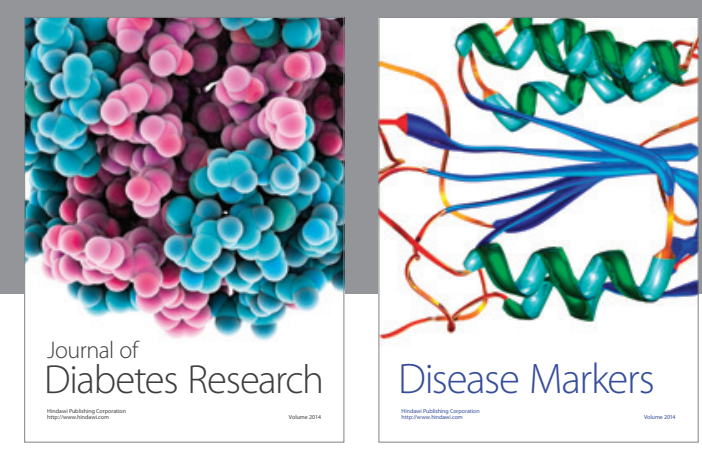

Disease Markers
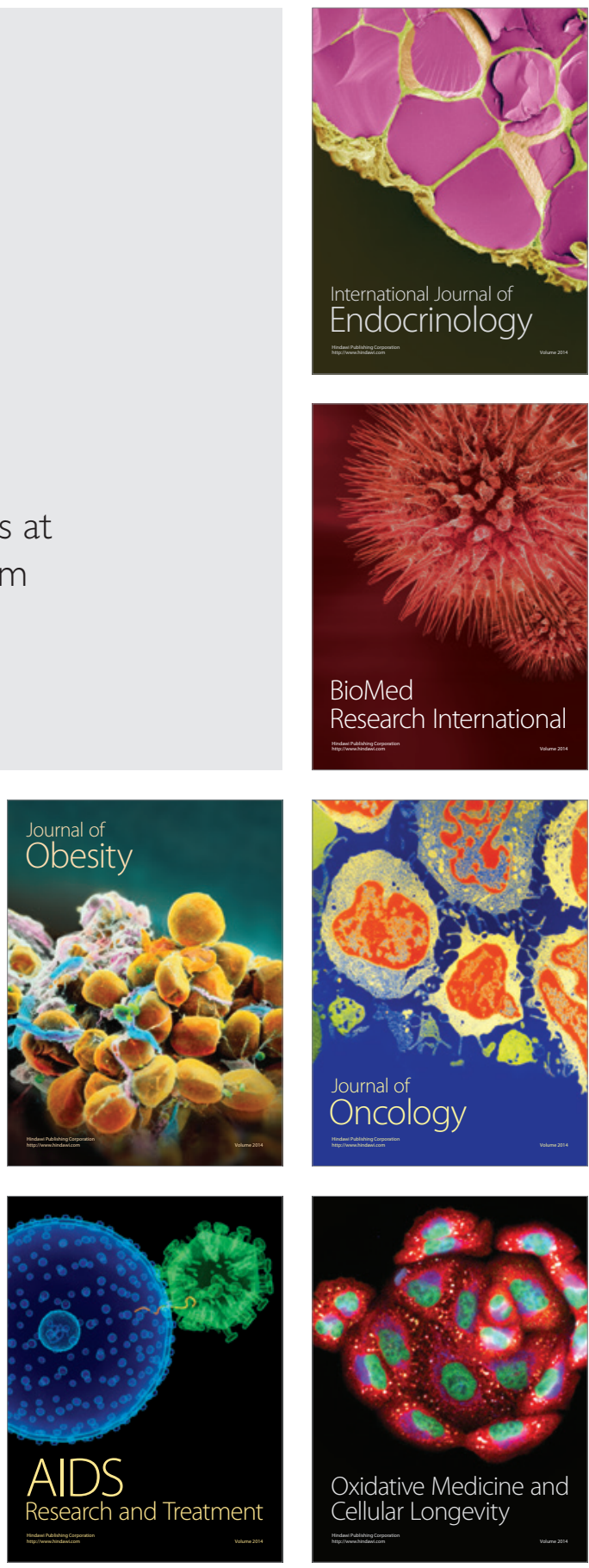